\section{Factores de riesgo para intento de suicidio en personas con trastorno depresivo en atención secundaria}

\author{
LILIAN SALVO ${ }^{1,2, a}$, JORDANA RAMÍREZ ${ }^{1, b}$, ANDREA CASTRO $^{3, \mathrm{~b}}$
}

\section{Risk factors for suicide attempts in people with depressive disorders treated in secondary health care}

Background: Depression is the most common psychiatric disorder in people with suicidal behavior. The knowledge of its risk factors should help to design preventive strategies. Aim: To describe suicidal behavior and risk factors for attempted suicide in people with major depressive disorders (MDD). Material and Methods: A 12-month follow-up study was conducted in 112 outpatients at three psychiatric care centers of Nuble, Chile, with baseline and quarterly assessments. Demographic, psychosocial and clinical factors as potential risk factors of suicide attempts, were assessed. A clinical interview with DSM-IV diagnostic criteria checklist, Hamilton Depression Scale and the List of Threatening Experiences and Multidimensional Scale of Perceived Social Support were applied. Results: Sixty seven percent of participants had suicidal ideation and $43.8 \%$ had attempted suicide. Suicide risk was significantly higher in participants with a single major depressive episode (odds ratio $[O R]=3.98 ; 95 \%$ confidence intervals $[C I]=1,29-12,32 p=0.02$ ) and those with previous suicide attempts $(O R=13.15 ; 95 \% C I=3,87-44.7 p<0.01)$. Young age, not having a partner, being unemployed, having a severe major depressive episode, having psychotic symptoms, having a personality disorder and being devoid of medical illness increased the risk of suicide attempts, but they did not reach statistical significance. Conclusions: Significant risk factors should be specially considered when designing suicide preventive strategies in patients with MDD.

(Rev Med Chile 2019; 147: 181-189)

Key words: Depression; Risk factors; Suicide, Attempted.
'Servicio de Psiquiatría,

Hospital Clínico Herminda Martín

Chillán, Chile.

${ }^{2}$ Facultad de Medicina,

Universidad Católica de

la Santísima Concepción.

Concepción, Chile.

${ }^{3}$ Universidad Diego Portales.

${ }^{a}$ Doctor en Salud Mental.

bPsicóloga.

Trabajo no recibió

financiamiento.

Los autores declaran no tener

conflcitos de interés.

Recibido el 11 de noviembre de 2018, aceptado el 17 de enero de 2019.

Correspondencia a:

Lilian Salvo G.

Bulnes 473, Chillán.

Isalvog@gmail.com

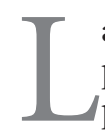

a depresión y la conducta suicida son importantes problemas de salud pública. La 1 prevalencia de 12 meses del trastorno depresivo mayor (TDM) fluctúa entre 2,2\% y $10,4 \%$ a nivel mundial ${ }^{1}$; en Chile, esta alcanzó a 5,7\% en la década de 1990-99, en una muestra de cuatro grandes regiones del país ${ }^{2}$ y a $18,4 \%$ en 2010 , en una muestra representativa a nivel nacional ${ }^{3}$. Por otro lado, según la Organización Mundial de la Salud $(\mathrm{OMS})^{4}$, las tasas de suicidio han aumentado en $60 \%$ en los últimos 45 años, constituyéndose en una de las tres primeras causas de muerte entre los 15 y 44 años y en la segunda causa entre los 10 y 29 años de edad. En el mundo, la tasa global de suicidio sería de 16 cada 100.000 habitantes. Respecto a las tentativas de suicidio, se estima que son hasta veinte veces más frecuentes que los suicidios consumados ${ }^{4}$. En Chile, las tasas de suicidio aumentaron progresivamente entre el año 2000 y 2011, especialmente en población adolescente. En el año 2015, la tasa global de mortalidad por esta causa fue de 9,23 por 100.000 habitantes ${ }^{5}$. 
Los estudios de autopsias psicológicas han demostrado que cerca de $90 \%$ de las víctimas de suicidio se encontraba cursando un trastorno psiquiátrico al momento de su muerte; siendo la depresión la enfermedad más frecuente, llegando a ser diagnosticada en aproximadamente en la mitad a dos tercios de los $\operatorname{casos}^{6,7}$. Una investigación de este tipo realizada en Chile reveló que 76,7\% de quienes se habían suicidado presentaban un episodio depresivo mayor (EDM) al momento de su muerte $^{8}$. Por otro lado, en pacientes depresivos se describen altas tasas de intentos de suicidio (alrededor de $40 \%$ tras el primer episodio) y de ideas suicidas de diversa gravedad (cercanas a $60 \%)^{9}$.

En base a estos antecedentes, se evidencia la importancia de estudiar factores de riesgo de conducta suicida en personas con TDM en nuestro medio, con el propósito de aportar información para estrategias preventivas.

El objetivo de este estudio es describir la suicidalidad y evaluar factores de riesgo de intento de suicidio en personas con TDM, en tratamiento en el nivel secundario del Servicio de Salud de Ñuble (SSÑ).

\section{Material y Método}

Se efectuó una investigación cuantitativa, no experimental, longitudinal, con seguimiento durante doce meses, con evaluación basal y cada 3 meses.

\section{Participantes}

El universo lo constituyeron todas las personas de 15 o más años de edad, con diagnóstico de TDM realizado por psiquiatra, que iniciaron tratamiento en los tres centros de atención secundaria del SSÑ, entre el 01/08/2013 y 31/07/2014. Los criterios de exclusión fueron trastorno bipolar, dependencia a sustancias en intoxicación o abstinencia, delirium, demencia, trastornos amnésicos, otros trastornos cognoscitivos, enfermedad médica o condición discapacitante que imposibilitara las evaluaciones y cambio de residencia fuera del SSÑ. A los que aceptaron participar se les efectuó entrevista clínica psiquiátrica con check list DSM-IV para reconfirmar diagnóstico. Quienes fueron ratificados con TDM ingresaron al estudio, alcanzándose una muestra de 112 participantes, $78,6 \%$ mujeres y $21,4 \%$ varones, de 15 a 79 años de edad, con promedio de 43,9 años (DE: 15,3). El seguimiento entre el 01/08/2013 y 31/07/2015 fue completado por 102 pacientes. El flujo de los participantes, tasa de respuesta y las características de las evaluaciones se encuentran publicadas ${ }^{10}$, ya que este estudio es parte de una investigación sobre el curso del TDM en el nivel secundario de atención del SSÑ.

\section{Variables}

Suicidalidad, definida como fenómeno suicida que incluye ideas, impulsos o conductas relacionados con el suicidio ${ }^{11}$. Fue evaluada por el Ítem 3: Suicidio, de la Escala de Depresión de Hamilton $(\mathrm{Ham}-\mathrm{D})^{12}$. En el análisis descriptivo se describirá cada una de las opciones: pensamientos de que la vida no merece la pena de ser vivida (VNP), deseos de estar muerto o pensamientos sobre la posibilidad de morirse (DEM), ideas o amenazas de suicidio e intento de suicidio. En el análisis correlacional se considerará como variable de resultado el intento de suicidio actual, entendido como el intento suicida realizado en el último mes.

Edad, Sexo, Nivel de Escolaridad, Estado Civil, Empleo y Residencia: recogidas con cuestionario sociodemográfico.

Eventos estresantes negativos: definidos como eventos que requieren un reajuste en las actividades cotidianas de los individuos y son percibidos como indeseables ${ }^{13,14}$; recogidos con el Listado de Eventos Estresantes (LEE), versión española ${ }^{15}$.

Apoyo social percibido: conceptualizado como la capacidad de sentirse perteneciente a una trama de relaciones personales que proveen estimación y ayuda ${ }^{16}$; medido por la Escala Multidimensional de Apoyo Social Percibido (EMASP) ${ }^{17}$ y operacionalizado por su puntaje total.

Severidad del EDM, Recurrencia del EDM (único o recurrente), Características melancólicas (presencia o ausencia) y Síntomas psicóticos (presencia o ausencia): definidas según DSM-IV ${ }^{18}$ y evaluada por entrevista psiquiátrica.

Enfermedad psiquiátrica comórbida Eje I y Trastorno de Personalidad: diagnosticadas por entrevista psiquiátrica según criterios DSM-IV ${ }^{18}$ y evaluada su presencia o ausencia.

Enfermedad médica comórbida: coocurrencia de una o más enfermedades médicas y TDM. Estos antecedentes fueron aportados por la(el) paciente y complementados con su historia clínica. Se analizó su presencia o ausencia. 
Intento de suicidio previo: intentos de suicidio realizados por el paciente en el último mes.

Suicidio en la familia: antecedente de suicidio en algún familiar. Consultado en entrevista psiquiátrica.

\section{Instrumentos}

Se aplicó:

Entrevista psiquiátrica con check list de criterios $D S M-I V$ : entrevista clínica basada en criterios diagnósticos del DSM-IV, de la American Psychiatric Association ${ }^{18}$. Estos criterios son ampliamente usados en clínica e investigación.

Ham-D (versión 17 items): evalúa el perfil sintomático y gravedad de la depresión ${ }^{12}$. Extensamente usada en clínica e investigación. Para evaluar la suicidalidad se empleó su Ítem 3 que consta de las siguientes opciones: 0: ausente, 1: VNP, 2: DEM, 3: ideas de suicidio o amenazas, 4: intentos de suicidio. En nuestro estudio Ham-D, obtuvo un coeficiente alfa de Cronbach de 0,77.

LEE: diseñado por Bruga et $\mathrm{al}^{14}$, inicialmente para investigar factores de riesgo de depresión. Actualmente se emplea en estudios de condiciones psiquiátricas, psicológicas y sociales. Consiste en un listado de 12 categorías de eventos estresantes negativos. Proporciona una puntuación total entre 0 y 12 eventos. Se empleó la adaptación española ${ }^{15}$ que evidenció adecuados índices de fiabilidad y validez.

EMASP: diseñada por Zimet et $\mathrm{al}^{17}$, compuesta por 12 ítems, recoge información del apoyo social percibido en familia, amigos y otros significativos. Sus opciones de respuestas corresponden a una escala de acuerdo entre 1 y 4 puntos. El puntaje total puede oscilar entre 0 y 48 puntos. En la investigación actual, el coeficiente alfa de Cronbach fue 0,87 .

\section{Procedimientos}

La investigación fue aprobada por el Comité de Ética del SSÑ. Se coordinó la obtención de registros en cada centro. Identificado el ingreso, se contactó personalmente al paciente y se invitó a participar. Quienes aceptaron, firmaron el consentimiento informado para el estudio y para obtener información de su historia clínica. Las personas se incorporaron al estudio a medida que fueron ingresando a tratamiento. Las evaluaciones fueron realizadas en dependencias del establecimiento de salud donde asisten a atención. Todos fueron entrevistados individualmente por psiquiatra en evaluación basal y por psicóloga en evaluaciones siguientes.

\section{Análisis de los datos}

Los datos fueron analizados por medio del programa estadístico SPSS V21. Se efectuó: a) análisis descriptivo a través de distribución de frecuencias, estadísticos de resumen y gráficos; b) análisis bivariado empleando la prueba $U$ de Mann-Whitney y prueba Chi-cuadrado de independencia; c) análisis multivariado por medio de regresión logística binaria. Se estableció nivel de significación estadística de 0,05.

\section{Resultados}

Del total de pacientes con TDM en tratamiento en el nivel secundario del SSÑ, al ingreso, 3,6\% (n: 4) no presentó suicidalidad; 4,5\% (n: 5) pensaba VNP; 15,2\% (n: 17) tenía DEM y 76,8\% (n: 86) presentaba ideas suicidas.

El 43,8\% (n: 49) había realizado intento de suicidio actual. De estos intentadores recientes, 79,6\% (n: 39) había cometido intentos suicidas previos. El 17\% (n: 19) tenía intentos de suicidio previos sin intento autolítico actual.

Considerando los intentadores previos y actuales, la edad promedio del primer intento suicida fue a los 30,2 años (DE: 13,9), con edad mínima: 11 años y máxima: 62 años. El promedio de intentos suicidas fue 2,8 (DE: 2,7), con mínimo: 1 y máximo: 15 intentos autolíticos.

Respecto al método suicida empleado en el intento actual, se reportaron: ingestión de sobre dosis de medicamentos: 61,2\% (n: 30), ahorcamiento: 16,3\% (n: 8), lesiones cortantes: el 14,3\% (n: 7) e ingestión de tóxicos: 4,1\% (n: 2). Un paciente $(2 \%)$ usó inyección de medicamentos (insulina) y otro (2\%) inhalación de tóxico (gas).

$\mathrm{El}$ antecedente de suicidio en la familia estuvo presente en $28 \%$ de los intentadores, ya sea previo o actual.

En la Figura 1 se presenta la evolución de la suicidalidad durante el seguimiento. El número de pacientes con VNP y DEM tiende a mantenerse. En cambio, la suicidalidad de mayor gravedad (ideas suicidas e intentos suicidas) disminuye progresivamente a través del tiempo de tratamiento, pero sin lograr que se sofoque totalmente en la 


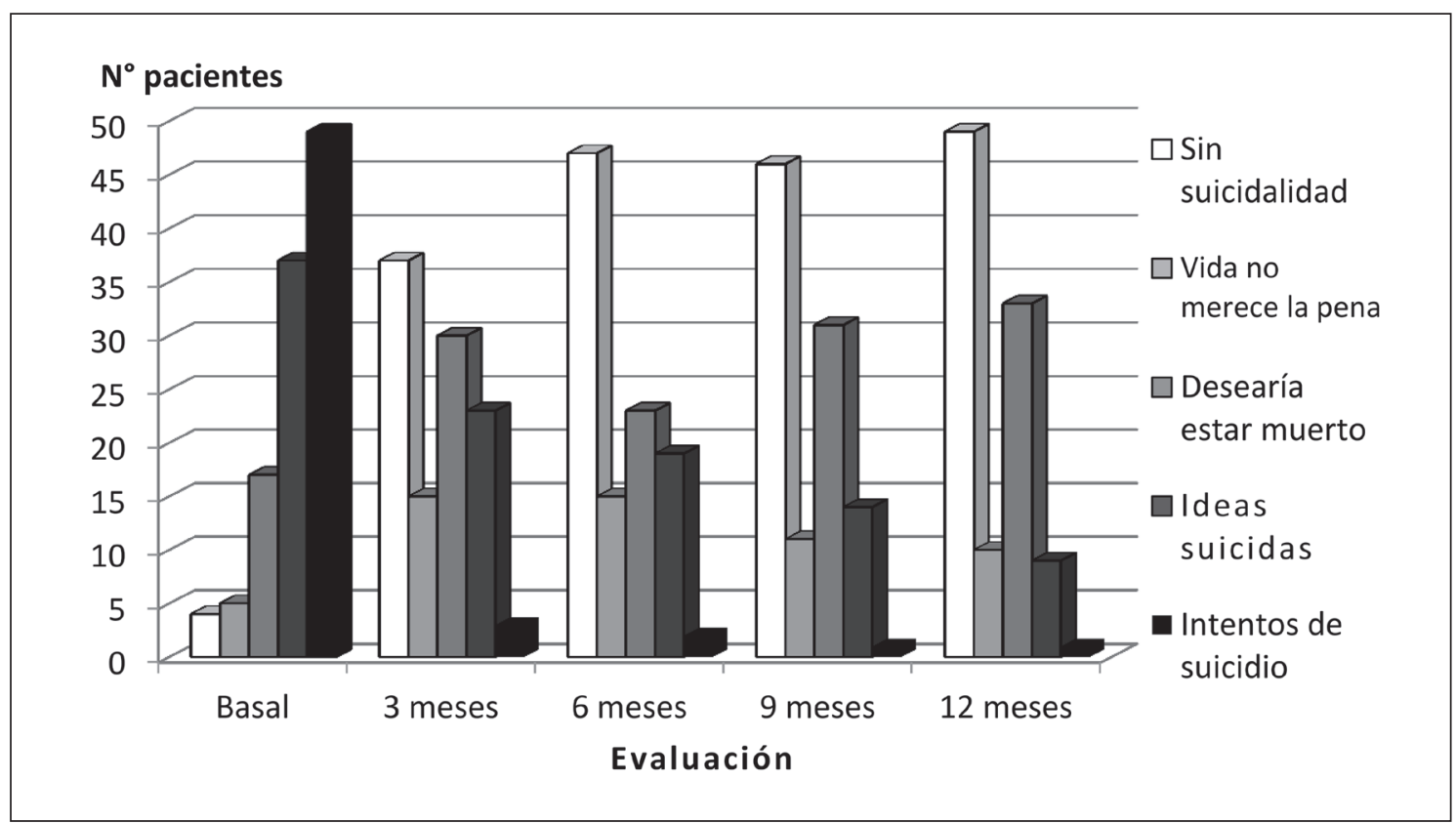

Figura 1. Evolución de la suicidalidad en pacientes con TDM tratado en el nivel secundario del Servicio de Salud de Ñuble, Chile (n: 112).

muestra. En los 12 meses hubo 7 intentos autolíticos, llevados a cabo por 5 pacientes: 3 intentaron una vez ( 2 a los tres meses y 1 a los 6 meses) y 2 hicieron dos nuevas tentativas (a los tres y nueve meses, y a los seis y doce meses, respectivamente). De estos 5 pacientes, 4 eran mujeres, de 41,5 años en promedio, 3 estaban sin trabajo, todas viviendo situaciones estresantes (promedio 2,8 eventos estresantes), todas percibían bajo apoyo social y 4 presentaban trastorno de personalidad. Ningún paciente se suicidó.

La relación entre variables demográficas y sociales con intento de suicidio actual puede observarse en la Tabla 1. Los resultados permiten plantear que solo la edad (menor edad) y el estado civil (solteros) se relacionan significativamente con intento de suicidio. Las personas con trabajo intentan menos suicidarse, pero sin significación estadística. El sexo, escolaridad y residencia, no se relacionaron con intento de suicidio actual. El número de eventos estresantes y el apoyo social percibido se relacionaron con la presencia de intento suicida actual en el sentido esperado, pero no alcanzaron significación estadística.

La relación entre variables clínicas e intento de suicidio actual se presenta en la Tabla 2. Se aprecia que la gravedad del EDM (grave), los EDM únicos, los síntomas psicóticos, el trastorno de personalidad, los intentos de suicidio previo y la ausencia de comorbilidad médica, se relacionan significativamente con intento de suicidio actual. Las características melancólicas, comorbilidad psiquiátrica eje I y el antecedente de suicidio en la familia, no se relacionaron con intento de suicidio actual.

Para evaluar los factores de riesgo, aquellas variables que resultaron significativas en su relación con intento de suicidio en el análisis bivariado fueron sometidas a análisis multivariado a través de regresión logística binaria. Los resultados se muestran en la Tabla 3. Este análisis revela que solo el EDM único y el antecedente de intento de suicidio previo resultaron ser estadísticamente significativas en su contribución a la presencia de intento de suicidio actual. Los pacientes con TDM con EDM único casi cuadruplican el riesgo comparados con los EDM recurrentes y los con intentos de suicidio previo tienen 13 veces más riesgo que quienes nunca han intentado suicidarse. Se encontró mayor riesgo de intento suicida en solteros(as) 
Tabla 1. Relación entre variables demográficas y psicosociales con intento de suicidio actual, en pacientes tratados en el nivel secundario del SSÑ (n: 112)

\begin{tabular}{|c|c|c|c|c|c|c|}
\hline \multirow[t]{2}{*}{$\begin{array}{l}\text { Características demográficas } \\
\text { y psicosociales }\end{array}$} & \multicolumn{2}{|c|}{$\begin{array}{l}\text { Con intento de } \\
\text { suicidio actual }\end{array}$} & \multicolumn{2}{|c|}{$\begin{array}{l}\text { Sin intento de } \\
\text { suicidio actual }\end{array}$} & \multirow[b]{2}{*}{ Coef } & \multirow[b]{2}{*}{$\mathbf{p}$} \\
\hline & $\mathbf{n}$ & $(\%)$ & $\mathbf{n}$ & (\%) & & \\
\hline$M(D S)$ & 38,4 & $(15,3)$ & 48,4 & $(13,9)$ & $-3,28^{a}$ & 0,000 \\
\hline Sexo & & & & & $1,35^{\mathrm{b}}$ & 0,246 \\
\hline Masculino & 13 & $(54,2)$ & 11 & $(45,8)$ & & \\
\hline Femenino & 36 & $(40,9)$ & 52 & $(59,1)$ & & \\
\hline Escolaridad & & & & & $0,65^{b}$ & 0,722 \\
\hline Básica & 16 & $(39,0)$ & 25 & $(61,0)$ & & \\
\hline Media & 19 & $(45,2)$ & 23 & $(54,8)$ & & \\
\hline Superior & 14 & $(48,3)$ & 15 & $(51,7)$ & & \\
\hline Estado civil & & & & & $8,47^{b}$ & 0,037 \\
\hline Soltero(a) & 22 & $(61,1)$ & 14 & $(38,9)$ & & \\
\hline Casado(a)/Conviviente & 18 & $(39,1)$ & 28 & $(60,9)$ & & \\
\hline Separado/Divorciado(a) & 8 & $(36,4)$ & 14 & $(63,6)$ & & \\
\hline Viudo(a) & 1 & $(12,5)$ & 7 & $(87,5)$ & & \\
\hline Empleo & & & & & $6,93^{b}$ & 0,140 \\
\hline Sin trabajo & 9 & $(47,4)$ & 10 & $(52,6)$ & & \\
\hline Con trabajo & 9 & $(27,3)$ & 24 & $(72,7)$ & & \\
\hline Jubilado & 4 & $(44,4)$ & 5 & $(55,6)$ & & \\
\hline Dueña de casa & 16 & $(47,1)$ & 18 & $(52,9)$ & & \\
\hline Estudiante & 11 & $(64,7)$ & 6 & $(53,3)$ & & \\
\hline Residencia & & & & & $0,05^{b}$ & 0,816 \\
\hline Urbano & 38 & $(43,2)$ & 50 & $(56,8)$ & & \\
\hline Rural & 11 & $(45,8)$ & 13 & $(54,2)$ & & \\
\hline n eventos vitales $M$ (DS) & 2,6 & $(0,20)$ & 2,5 & $(0,19)$ & $-0,34^{a}$ & 0,737 \\
\hline Apoyo social & 24,7 & $(1,34)$ & 25,4 & $(1,07)$ & $-0,42^{a}$ & 0,675 \\
\hline
\end{tabular}

aPrueba U de Mann-Whitney. ${ }^{\text {bPrueba }} \chi^{2}$.

(el doble que los casados), en EDM graves (tres veces superior a episodios moderados), síntomas psicóticos (el doble que sin psicosis) y trastorno de personalidad ( 1,4 veces superior a los que no lo padecen), pero estas características no alcanzaron significación estadística.

\section{Discusión}

Tal como se ha demostrado en múltiples investigaciones, nuestro estudio pone nuevamente en evidencia la alta suicidalidad presente en pacientes con $\mathrm{TDM}^{9}$ (76,8\% de ideas suicidas y $43,8 \%$ de intento de suicidio actual).
Teniendo presente que la presencia de un trastorno depresivo ya es un potente predictor para suicidalidad, identificar factores de riesgo de intento suicida en esta población cobra mayor relevancia, ya que su detección permite identificar grupos o personas con mayor probabilidad de conducta suicida, reducir el riesgo y diseñar estrategias que consideren tales factores ${ }^{9}$. Con ese objetivo realizamos nuestro análisis, encontrando solo como factor de riesgo significativo la presencia de EDM único, es decir, un primer EDM y el antecedente de intentos de suicido previos. Como en otros estudios, este último resultó ser un potente factor de riesgo ${ }^{9,19}$. 
Tabla 2. Relación entre variables clínicas e intento de suicidio actual, en pacientes tratados en el nivel secundario del SSÑ (n: 112)

\begin{tabular}{|c|c|c|c|c|c|c|}
\hline \multirow[t]{2}{*}{ Características clínicas } & \multicolumn{2}{|c|}{ Con intento de suicidio } & \multicolumn{2}{|c|}{ Sin intento de suicidio } & \multirow[b]{2}{*}{ Coef } & \multirow[b]{2}{*}{$\mathbf{p}$} \\
\hline & & (\%) & & $(\%)$ & & \\
\hline Severidad EDM & & & & & 4,04 & 0,044 \\
\hline Moderado & 4 & $(22,2)$ & 14 & $(77,8)$ & & \\
\hline Grave & 45 & $(47,9)$ & 49 & $(52,1)$ & & \\
\hline Recurrencia & & & & & 4,26 & 0,039 \\
\hline EDM único & 25 & $(55,6)$ & 20 & $(44,4)$ & & \\
\hline EDM recurrente & 24 & $(35,8)$ & 43 & $(64,2)$ & & \\
\hline C. Melancólicas & & & & & 0,46 & 0,495 \\
\hline $\sin$ & 28 & $(41,2)$ & 40 & $(58,8)$ & & \\
\hline Con & 21 & $(47,7)$ & 23 & $(52,3)$ & & \\
\hline Síntomas psicóticos & & & & & 4,59 & 0,032 \\
\hline $\sin$ & 28 & $(36,8)$ & 48 & $(63,2)$ & & \\
\hline Con & 21 & $(58,3)$ & 15 & $(41,7)$ & & \\
\hline Co-psiquiátrica EJE I & & & & & 0,78 & 0,376 \\
\hline $\sin$ & 29 & $(47,5)$ & 32 & $(52,5)$ & & \\
\hline Con & 20 & $(39,2)$ & 31 & $(60,8)$ & & \\
\hline Trastorno personalidad & & & & & 5,12 & 0,024 \\
\hline $\sin$ & 11 & $(28,9)$ & 27 & $(71,1)$ & & \\
\hline Con & 38 & $(51,4)$ & 36 & $(48,6)$ & & \\
\hline Intento suicida previo & & & & & 26,98 & 0,000 \\
\hline $\sin$ & 10 & $(18,5)$ & 44 & $(81,5)$ & & \\
\hline Con & 39 & $(67,2)$ & 19 & $(32,8)$ & & \\
\hline Suicidio en la familia & & & & & 2,44 & 0,296 \\
\hline $\sin$ & 36 & $(45,0)$ & 44 & $(55,0)$ & & \\
\hline Con & 12 & $(46,2)$ & 14 & $(53,8)$ & & \\
\hline Comorbilidad médica & & & & & 4,68 & 0,031 \\
\hline $\sin$ & 22 & $(57,9)$ & 16 & $(42,1)$ & & \\
\hline Con & 27 & $(36,5)$ & 47 & $(63,5)$ & & \\
\hline
\end{tabular}

Se empleó Prueba $\chi^{2}$ para el análisis de relación de cada variable. EDM: Episodio depresivo mayor. C: Características. Co: Comorbilidad.

Otros factores, ya evidenciados por otros autores ${ }^{19-21}$, tales como menor edad, estado civil soltero, EDM grave, síntomas psicóticos y trastorno de personalidad, se relacionaron con intento de suicido actual, aumentando su riesgo, pero no alcanzaron significación estadística. Estimamos que probablemente este hecho se debe a que la muestra fue numéricamente insuficiente.

Respecto al EDM único como factor de riesgo, las estrategias deberían estar dirigidas a identificar grupos o personas con riesgo de hacer un primer EDM para reducir su incidencia. En este sentido, a nivel internacional existen programas de prevención universal y selectiva que han demostrado resultados beneficiosos ${ }^{22-26}$. El desafío es implemen- tarlos en nuestro país. A nivel nacional, además, se requiere mejorar la educación e intervención a nivel de la comunidad para consulta precoz. En salud aún se debería trabajar en mejorar el acceso a consultas y efectuar capacitación continua a los distintos profesionales para pesquisar sintomatología depresiva. En investigación, en Chile se han realizado estudios sobre factores de riesgo para el inicio de la depresión, proporcionando evidencia para futuras estrategias preventivas ${ }^{27,28}$. Por otro lado, ya instalado el EDM, su diagnóstico y tratamiento oportuno y adecuado disminuye el riesgo de suicidalidad ${ }^{29}$. En estos pacientes con EDM se debería prestar atención a factores que se relacionan con conducta suicida (algunos de 
Tabla 3. Riesgos de presentar intento de suicidio, en pacientes con TDM tratados en el nivel secundario del SSÑ

\begin{tabular}{|c|c|c|c|c|c|}
\hline \multirow[t]{3}{*}{ Predictores } & & \multicolumn{4}{|c|}{ Intento de suicidio actual } \\
\hline & & \multirow[t]{2}{*}{$\mathbf{p}$} & \multirow[t]{2}{*}{ OR } & \multicolumn{2}{|c|}{ IC $95 \%$} \\
\hline & & & & Inf & Sup \\
\hline \multicolumn{2}{|l|}{ Edad } & 0,295 & 0,977 & 0,936 & 1,020 \\
\hline \multirow[t]{4}{*}{ Estado civil } & Casado(a) & & 1 & & \\
\hline & Soltero(a) & 0,218 & 2,582 & 0,572 & 11,662 \\
\hline & Separado/divorciado(a) & 0,689 & 0,750 & 0,183 & 3,068 \\
\hline & Viudo(a) & 0,622 & 0,541 & 0,047 & 6,220 \\
\hline \multirow[t]{2}{*}{ Severidad del episodio depresivo } & Moderado & & 1 & & \\
\hline & Grave & 0,191 & 2,827 & 0,596 & 13,404 \\
\hline Recurrencia & $\begin{array}{l}\text { Único } \\
\text { Recurrente }\end{array}$ & 0,017 & $\begin{array}{c}3,978 \\
1\end{array}$ & 1,285 & 12,317 \\
\hline \multirow{2}{*}{ Síntomas psicóticos } & $\sin$ & & 1 & & \\
\hline & Con & 0,182 & 2,131 & 0,701 & 6,478 \\
\hline \multirow[t]{2}{*}{ Trastorno de personalidad } & $\sin$ & & 1 & & \\
\hline & Con & 0,551 & 1,401 & 0,462 & 4,245 \\
\hline \multirow[t]{2}{*}{ Intento de suicidio previo } & $\operatorname{Sin}$ & & 1 & & \\
\hline & Con & 0,000 & 13,146 & 3,866 & 44,700 \\
\hline \multirow[t]{2}{*}{ Comorbilidad médica } & $\sin$ & & 1 & & \\
\hline & Con & 0,258 & 0,466 & 0,124 & 1,747 \\
\hline
\end{tabular}

Análisis multivariado con regresión logística binaria.

los cuales fueron corroborados por nuestro estudio), como sexo femenino, menor edad, estar soltero, desventajas socioeconómicas, mayor gravedad de la depresión, síntomas psicóticos, trastorno de personalidad, trastorno por consumo de sustancias, estrés postraumático, trastorno de ansiedad, impulsividad, desesperanza, intentos suicidas previos, hospitalizaciones previas e historia familiar de trastornos psiquiátricos ${ }^{19-21}$. Estos factores pueden aumentar el riesgo de reiteración de tentativas autolíticas como lo observado durante nuestro seguimiento, donde 5 pacientes volvieron a realizar intentos suicidas; ellos en su mayoría eran mujeres, sin trabajo, con eventos estresantes, menor apoyo social y con trastorno de personalidad.

Para abordar el antecedente de intento de suicidio como factor de riesgo, existen propuestas internacionales como el Programa de Prevención del Suicidio de la OMS, dirigido a grupos sociales y a profesionales ${ }^{4}$. En nuestro país se cuenta con un programa ministerial de prevención del suicidio ${ }^{30}$ e importantes estrategias preventivas en algunas regiones, como las desarrolladas por la Alianza
Chilena contra la Depresión en la Región de los Ríos $^{31}$ y el Programa Radar en la Región de Aysén ${ }^{32}$. En el ambiente clínico, para ayudar a prevenir la ocurrencia de intentos de suicidio cobra relevancia la búsqueda de factores de riesgo, especialmente los trastornos psiquiátricos y las ideas suicidas. En atención primaria, secundaria y terciaria se debería tener presente el consultar por ideas suicidas en todo paciente con síntomas psicopatológicos, especialmente depresivos, en pacientes con condiciones médicas asociadas a riesgo suicida, en grupos de edad con mayor riesgo (adolescentes, adultos mayores) y en quienes estén viviendo situaciones de adversidad socioeconómica.

Este estudio cuenta con limitaciones que dicen relación especialmente con el tamaño de la muestra y que no es representativo de toda la realidad nacional. Habría sido deseable además evaluar señales de alarma de intento de suicidio.

Agradecimientos: Se agradece a Carlos Parra, Manuel Cifuentes, Paola Acevedo, Marcela Díaz, Mitza Ormazabal, Ivonne Guerra, Nicol Navarrete y Verónica Bravo por su colaboración en la in- 
vestigación sobre el curso del trastorno depresivo mayor en el nivel secundario de atención, de la cual es parte este estudio.

\section{Referencias}

1. Bromet EJ, Andrade L, Hwang I, Sampsom N, Alonso J, De Girolamo G, et al. Cross-national epidemiology of DSM-IV major depressive episode. BMC Medicine 2011; 9: 90-105.

2. Vicente B, Kohn R, Rioseco P, Saldivia S, Levav I, Torres S. Lifetime and 12-Month Prevalence of DSM-III-R Disorders in the Chile Psychiatric Prevalence Study. Am J Psychiatry 2006; 163: 1362-70.

3. Markkula N, Zitko P, Peña S, Margozzini P, Retamal P. Prevalence, trends, correlates and treatment of depression in Chile in 2003 to 2010. Soc Psychiatry Psychiatr Epidemiol 2017; 52 (4): 399-409.

4. OMS. Prevención del suicidio. Disponible en http:// www.who.int/mental_health/prevention/suicide/suicideprevent/es/ (Consultado el 13 de noviembre de 2017).

5. Ministerio de Salud de Chile. Series y gráficos de mortalidad. Disponible en www.deis.cl/series-y-graficos-de-mortalidad/ (Consultado el 13 de noviembre de 2017).

6. Arsenault-Lapierre G, Kim C, Turecki G. Psychiatric diagnoses in 3275 suicides: a meta-analysis. BMC Psychiatry 2004; 4: 37.

7. Bradvik L, Mattisson C, Bogren M, Nettelbladt P. Mental desordersin suicide and undetermined death in the Lundly Study. The contribution of severe depression and alcohol dependence. Arch Suicide Res 2010; 14 (3): 266-75.

8. Gómez A, Opazo R, Levi R, Gómez M, Ibáñez C, Núñez C. Autopsias psicológicas de treinta suicidios en la IV Región de Chile. Rev Chil Neuro-Psiquiat 2014; 52 (1): 9-19.

9. Gómez A, Ibáñez C. Factores de riesgo de suicidio: una visión general. En: Gómez A, Silva H, Amon R, editores, El suicidio. Teoría y clínica. Santiago, Chile: Editorial Mediterráneo Ltda.; 2018. p. 28-43.

10. Salvo L, Saldivia S, Parra C, Cifuentes M, Bustos C, Acevedo P, et al. Predictores de remisión del trastorno depresivo mayor en tratamiento en el nivel secundario de atención. Rev Med Chile 2017; 145: 1514-24.

11. Sheehan D, Giddens J. Suicidality: A Roadmap for assessment and treatment. Tampa, FL, US: Harm Research Press, 2015.

12. Hamilton M. A rating scale for depression. J Neurol Neurosurg Psychiatry 1960; 23: 56-62.
13. Monroe SM. Modern approaches to conceptualizing and measuring human life stress. Annu Rev Clin Psychol 2008; 4: 33-52.

14. Brugha TS, Cragg D. The List of Threatening Experiences: the reliability and validity of a brief life events questionnaire. Acta Psychiatr Scand 1990; 82: 77-81.

15. Motrico E, Moreno-Küstner B, De Dios Luna J, Torres-González F, King M, Nazareth I, et al. Psychometric properties of the List of Threatening Experiences - LTE and its association with psychosocial factors and mental disorders according to different scoring methods. J Affect Disord 2013; 150 (3): 931-40.

16. Uchino B, Cacioppo J, Kiecolt-Glasser J. The relationship between social support and physiological processes: a review with emphasis on underlying mechanism and implications for health. Psychol Bull 1996; 119: 488-531.

17. Zimet G, Dahlem N, Zimet S, Farley G. Multidimensional Scale of Perceived Social Support. J Pers Assess 1988; 52 (1): 30-41.

18. Asociación Americana de Psiquiatría: Manual Diagnóstico y Estadístico de los Trastornos Mentales (DSM-IVTR). Barcelona: Masson; 2009.

19. Hawton K, Casañas C, Haw C, Saunders K. Risk factors for suicide in individuals with depression: A systematic review. J Affect Disord 2013; 147: 17-28.

20. Lewitzka U, Spirling S, Ritter D, Smolka M, Goodday S, Bauer M, et al. Suicidal Ideation vs. suicide attempts: Clinical and psychosocial profile differences among depressed patients. A Study on personality traits, psychopathological variables, and sociodemographic factors in 228 patients. J Nerv Ment Dis 2017; 205: 361-71.

21. Cavalcante I, Mwangi B, Cao B, Hamilton J, Wu M, Yang $\mathrm{X}$, et al. Identifying a clinical signature of suicidality among patients with mood disorders: a pilot study using a machine learning approach. J Affect Disord 2016; 193: 109-16.

22. Organización Mundial de la Salud. Prevención de los trastornos mentales: intervenciones efectivas y opciones de políticas: informe compendiado. Ginebra: OMS; 2004.

23. Merry SN, Hetrick SE, Cox GR, Brudevold-Iversen T, Bir JJ, McDowell H. Psychological and educational interventions for preventing depression in children and adolescents. Cochrane Database Syst Rev 2011: CD003380.

24. Forsman AK, Schierenbeck I, Wahlbeck K. Psychosocial interventions for the prevention of depression in older adults: systematic review and meta-analysis. J Aging Health 2011; 23: 387-416.

25. Van der Waerden JE, Hoefnagels C, Hosman CM. Psy- 
chosocial preventive interventions to reduce depressive symptoms in low-SES women at risk: a meta-analysis. J Affect Disord 2011; 128: 10-23.

26. Stice E, Shaw H, Bohon C, Marti CN, Rohde P. A meta-analytic review of depression prevention programs for children and adolescents: factors that predict magnitude of intervention effects. J Consult Clin Psychol 2009; 77: 486-503.

27. Saldivia S, Vicente B, Marston L, Melipillán R, Nazareth I, Bellon-Saameno J, et al. [Development of an algorithm to predict the incidence of major depression among primary care consultants]. Rev Med Chile 2014; 142: 323-9.

28. Vicente B, Rojas R, Saldivia S, Pérez C, Melipillán R, Hormazabal $\mathrm{N}$, et al. Determinantes biopsicosociales de depresión en pacientes atendidos en Centros de
Atención Primaria de Concepción, Chile. Rev Chil Neuro-Psiquiat 2016; 54 (2): 102-12.

29. Retamal P. Depresión y suicidio. En: Gómez A, Silva H, Amon R, editores, El suicidio. Teoría y clínica. Santiago, Chile: Editorial Mediterráneo Ltda.; 2018. p. 83-91.

30. Ministerio de Salud. Programa nacional de prevención del suicidio. Orientaciones para su implementación. Santiago, Chile: Minsal; 2013.

31. Alianza Chilena Contra la Depresión. Disponible en http://www.achid.cl (Consultado el 21 de septiembre de 2018).

32. Bustamante F, Urquidia C, Florenzano R, Barrueto C, De los Hoyos J, Ampuero K, et al. El programa RADAR para la prevención del suicidio en adolescentes de la región de Aysén, Chile: resultados preliminares. Rev Chil Pediatr 2018; 89 (1): 145-8. 